09

\title{
CuBr-лазер большого объема с возможностью непрерывного управления энергией генерации
}

\author{
(c) М.В. Тригуб, В.О. Троицкий \\ Институт оптики атмосферы им. В.Е. Зуева СО РАН, Томск, Россия \\ E-mail: trigub@iao.ru
}

Поступило в Редакцию 5 ноября 2019 г.

В окончательной редакции 5 ноября 2019 г.

Принято к публикации 29 января 2020 г.

\begin{abstract}
Представлены результаты разработки и исследования макета активного элемента на парах бромида меди с непрерывным управлением энергией каждого импульса излучения. Отличительными особенностями являются реализация указанного режима для газоразрядной трубки большого объема (более 1.51) и формирование излучения неустойчивым резонатором. При этом использовалась водородсодержащая добавка для модификации кинетики плазмы с целью увеличения мощности излучения. Управление осуществлялось за счет использования дополнительного импульса предионизации. Получена зависимость мощности генерации на каждой линии от энергии импульса предионизации и временно́й задержки между этим импульсом и импульсами возбуждения. Показано, что в разработанном макете определяющим фактором в управлении генерацией является заселение метастабильных уровней атомов меди.
\end{abstract}

Ключевые слова: активные среды на парах металлов, управление генерацией, $\mathrm{CuBr}$-лазер, функциональные преобразователи.

DOI: 10.21883/PJTF.2020.08.49308.18095

Отличительным преимуществом активных сред на самоограниченных переходах атомов металлов является возможность преобразования оптических сигналов с сохранением заданного распределения интенсивности различных спектральных диапазонов [1]. Эта особенность в совокупности с высокой спектральной яркостью указанных сред позволяет формировать изображения объектов, экранированных широкополосной засветкой [2]. Разработка и исследование активных сред на парах металлов с целью улучшения их передаточных и частотноэнергетических характеристик остаются актуальной задачей, успешное решение которой позволит создать скоростные усилители яркости видимого и ближнего ИК-диапазонов спектра [3]. При этом использование специальных решений по созданию паров рабочего вещества позволяет получать генерацию даже в тугоплавких металлах [4].

Несмотря на длительный этап изучения характеристик таких сред, оптимизации режимов их работы и улучшения частотно-энергетических и эксплуатационных характеристик, остается ряд нерешенных задач, в частности создание методов и средств, обеспечивающих непрерывное (поимпульсное) управление энергетическими и усилительными параметрами указанных сред в режиме реального времени. Высокая инерционность активной среды, обусловленная термодинамическими процессами, не позволяет осуществлять оперативное управление параметрами каждого импульса излучения за счет изменения энергии отдельного импульса возбуждения. Для таких сред целесообразно осуществлять управление за счет подачи дополнительного импульса, что было продемонстрировано для лазеров на парах чистой меди в работе [5]. Использование дополнительного импульса предионизации для оперативного управления параметрами излучения в лазере на парах бромида меди среднего объема при работе в оптимальном частотном диапазоне (десятки $\mathrm{kHz}$ ) рассматривается в [6]. Сообщается о получении мощности $1 \mathrm{~W}$ для газоразрядной трубки объемом $1131 \mathrm{~cm}^{3}$, что указывает на неоптимальный режим функционирования активного элемента, например малую концентрацию $\mathrm{CuBr}$. В работе мощность источника накачки ограничивается $1 \mathrm{~kW}$, что для газоразрядной трубки (ГРТ) такого объема является недостаточным. В [7] показана возможность непрерывного управления энергией лазера на парах бромида меди малого объема с помощью магнитного поля.

Отличительной особенностью лазеров н парах металлов большого объема является возможность использования неустойчивого резонатора, что позволяет формировать излучения с малой расходимостью. В этом случае очень важна стабильность пространственных характеристик усиления в среде, которая влияет на формируемое излучение [8]. Кроме того, этот большой объем позволяет получать большие мощности излучения при оптимальном тепловом режиме и наличии добавки $\mathrm{HBr}$ [9].

Целью настоящей работы является разработка системы управления параметрами активной среды на парах бромида меди большого объема, а также исследование возможностей непрерывного управления энергией излучения, сформированного в неустойчивом резонаторе, при оптимальном тепловом режиме.

Для проведения исследований был разработан макет, структурная схема которого представлена на рис. 1. 


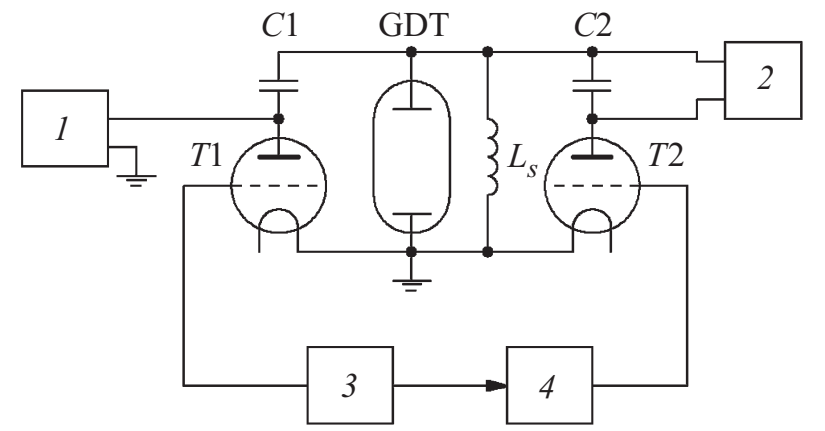

Рис. 1. Схема экспериментальной установки. Пояснения в тексте.

Импульсы накачки и предионизации формировались двумя независимыми источниками (1 и 2), работа которых согласовывалась схемой синхронизации (3). Каждый источник представлял собой схему с импульсным зарядом рабочей емкости. Рабочие емкости разряжались на ГРТ через тиратроны ТГИ1-1000/25 и ТГИ1-500/16.

Джиттер между импульсами возбуждения и предионизации не превышал $100 \mathrm{~ns}$ в условиях эксперимента. Длина и диаметр активной зоны ГРТ составляли 90 и $5 \mathrm{~cm}$ соответственно (объем составлял $1766.3 \mathrm{~cm}^{3}$ ). Излучение формировалось неустойчивым резонатором с боковым выводом. Стационарный тепловой режим активной зоны, неизменные концентрации рабочего вещества $(\mathrm{CuBr})$ и водородсодержащей добавки ( $\mathrm{HBr})$ обеспечивались трехканальным термостабилизатором. Измерение электрических характеристик разряда осуществлялось с помощью датчика тока Pearson Current Monitor 8450 и датчика напряжения Tektronics P6015A. Для регистрации импульсов излучения применялись фотодиоды DET 10A/M. Импульсы фиксировались с помощью осциллографа LeCroy WJ-324. Измерение мощности излучения проводилось с помощью Ophir 30C-SH.

Источник 1 формировал высоковольтные импульсы накачки с частотой следования импульсов $10.5 \mathrm{kHz}$, напряжение на накопительном конденсаторе $C 1$ емкостью $1000 \mathrm{pF}$ было равно $11 \mathrm{kV}$, мощность, потребляемая от сети, составляла $1.5 \mathrm{~kW}$. В рамках эксперимента энергия импульса возбуждения оставалась постоянной и составляла $60.5 \mathrm{~mJ}$, что соответствует удельному энерговкладу $29.2 \mu \mathrm{J} / \mathrm{cm}^{3}$. Средняя мощность излучения составляла $5 \mathrm{~W}$ при возбуждении активного элемента источником 1 (без импульсов предионизации). Данная мощность была максимальной для параметров возбуждения, используемых в эксперименте. Варьирование теплового режима активного элемента, концентрации $\mathrm{CuBr}$ и $\mathrm{HBr}$ приводило к уменьшению средней мощности генерации. При оценке влияния импульса предионизации, формируемого источником 2, параметры импульса возбуждения оставались неизменными. Варьирование энергии импульсов предионизации в диапазоне от 0.5 до $10 \mathrm{~mJ}$ обеспечивается за счет использования различных емкостей $C 2$ и изменения максимального напряжения, до которого они заряжаются. Время задержки $(\tau)$ между импульсами возбуждения и предионизации плавно изменялось в диапазоне от 0 до $25 \mu \mathrm{s}$ с помощью системы управления (3) и линии задержки (4). В рамках работы зависимость мощности генерации лазера, нормированной на максимальное значение, от временно́го сдвига между импульсами возбуждения и предионизации будем называть регулировочной характеристикой. На рис. 2, a приведены осциллограммы импульсов накачки и генерации при различном значении временно́го сдвига $(5,10$ и $25 \mu \mathrm{s}$ ). Емкость $C 2$ составляла $1500 \mathrm{pF}$, амплитуда напряжения на емкости была равна $3 \mathrm{kV}$, что соответствует энергии $6.75 \mathrm{~mJ}$.

Регулировочные характеристики для каждой линии излучения (510.6 и $578.2 \mathrm{~nm})$ показаны на рис. $2, b$. Для выделения отдельной спектральной линии использовался метод, представленный в работе [7], когда спектральный фильтр располагался внутри резонатора, что обеспечивает минимальные потери. Нормирование проводилось на значение мощности при отсутствии импульсов предионизации. Как видно из полученных зависимостей, полностью подавить генерацию удается при задержках в диапазоне $1-2 \mu \mathrm{s}$ с учетом аппаратного джиттера. Излучение на желтой линии генерации $(578.2 \mathrm{~nm})$ начинает появляться при сдвиге $2 \mu \mathrm{s}$, тогда как генерация на зеленой линии $(510.6 \mathrm{~nm})$ начинает появляться только при временно́м сдвиге более $4 \mu \mathrm{s}$. Дальнейшее увеличение задержки между импульсами приводит практически к линейному росту мощности генерации на обеих линиях излучения. Восстановление генерации до исходного значения наступает при задержке $24 \mu \mathrm{s}$. Дальнейшее увеличение задержки никак не сказывается на мощности генерации, которая равна исходному значению $(5 \mathrm{~W})$.

Ослабление генерации при вводе дополнительного импульса может быть связано с двумя факторами [10]. Вопервых, это изменение предимпульсной концентрации электронов, что приводит к изменению электрических параметров разрядного промежутка. Во-вторых, дополнительное заселение метастабильных уровней атомов металла импульсом предионизации, в результате чего инверсия уменьшается. Изменение предимпульсной концентрации электронов в результате подачи на ГРТ импульса предионизации приводит к изменению проводимости плазмы. Скорость нарастания, амплитудные значения и временна́я задержка между импульсами тока и напряжения возбуждения практически не изменяются при изменении временно́го сдвига между импульсами источников 1 и 2. Это видно на осциллограммах, представленных на рис. 2, $a$. Исходя из этого можно заключить, что в условиях наших экспериментов подавление генерации происходит преимущественно за счет изменения заселенностей метастабильных уровней. Косвенно об этом свидетельствует и поведение мощности генерации на желтой и зеленой линиях излучения. Метастабильный уровень $D 2$ (нижний рабочий уровень перехода $578.2 \mathrm{~nm}$ ) находится выше, чем $D 1$ (нижний 

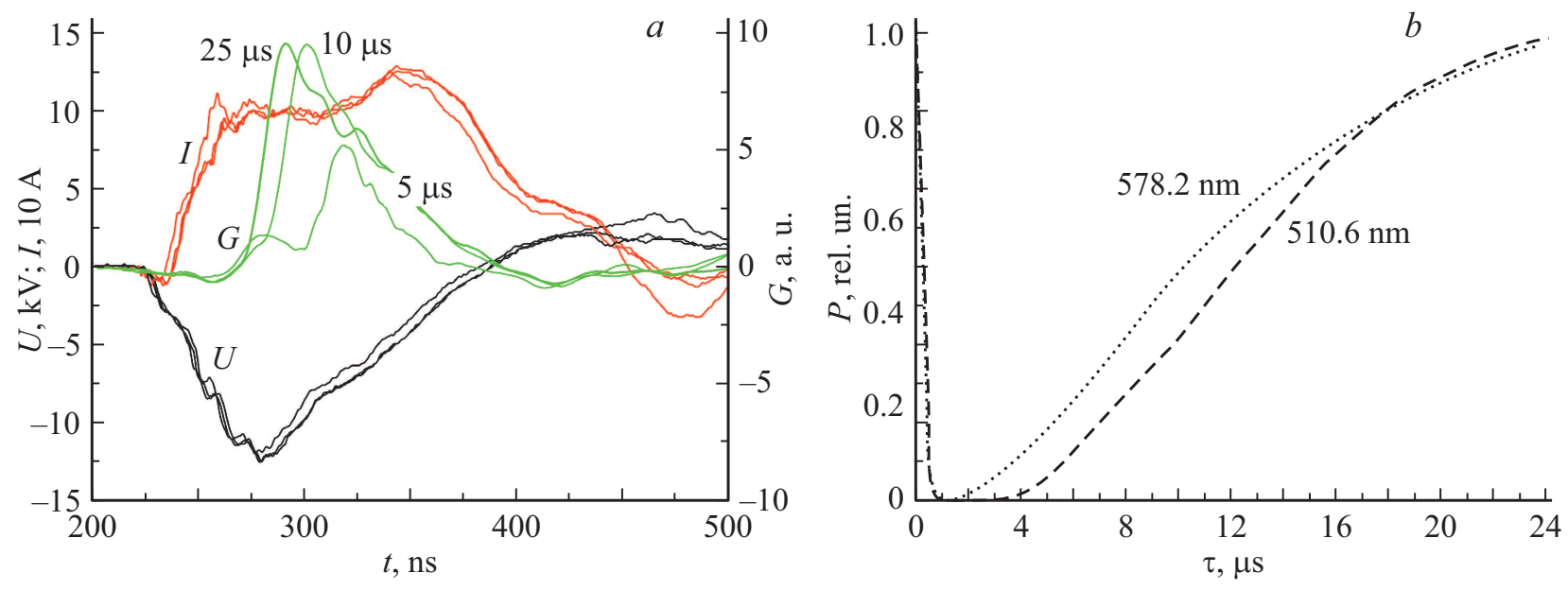

Рис. 2. Осциллограммы импульсов $(a)$ напряжения $(U)$, тока $(I)$ и генерации $(G)$ и регулировочная характеристика $(b)$.

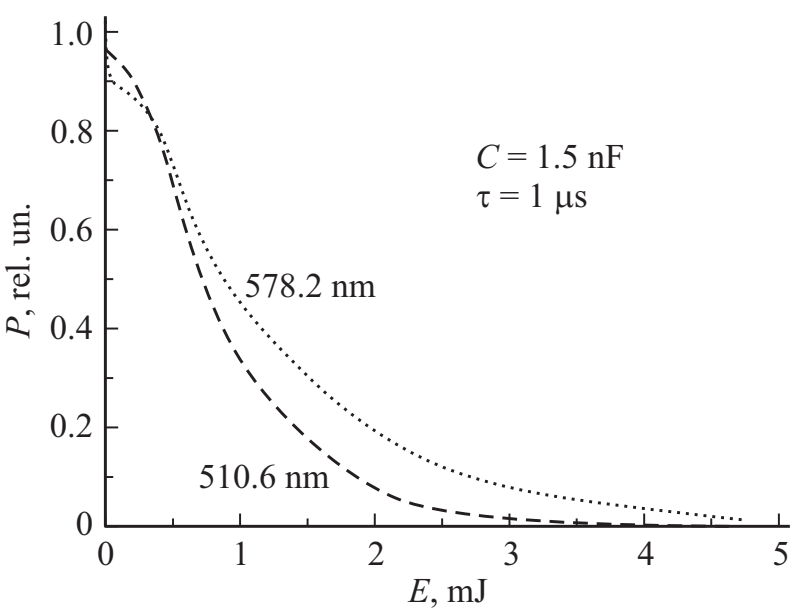

Рис. 3. Зависимость мощности излучения от энергии импульса гашения при задержке $1 \mu \mathrm{s}$.

рабочий уровень перехода $510.6 \mathrm{~nm})$. Очевидно, что заселенность уровня $D 2$ будет ниже, чем заселенность уровня $D 1$ по окончании импульса предионизации. Увеличение временно́й задержки обеспечивает возможность релаксации этих уровней к моменту подачи импульса возбуждения, что и приводит к восстановлению излучения. Инверсия заселенности для желтой линии начинает наблюдаться при меньших задержках, в то время как для создания инверсии на зеленой линии требуется большее время. Известно [1], что излучение на линии $510.6 \mathrm{~nm}$ формируется раньше и занимает основную часть переднего фронта импульса генерации, а излучение $578.2 \mathrm{~nm}$ формируется спустя $10-15 \mathrm{~ns}$. При вводе импульса предионизации наблюдается подавление переднего фронта импульса генерации (рис. $2, a$ ), что особенно хорошо видно при задержке $5 \mu \mathrm{s}$. В этом случае излучение на линии $510.6 \mathrm{~nm}$ практически отсутствует (согласно рис. $2, b)$, а излучение на линии $578.2 \mathrm{~nm}$ составляет $3 \%$ от максимального значения.
Очевидно, что на мощность генерации влияет не только задержка между импульсами, но и параметры импульса предионизации. Экспериментально получена зависимость мощности излучения на каждой спектральной линии от энергии импульса предионизации (рис. 3). При измерении мощности генерации задержка между импульсами накачки и предионизации не изменялась и составляла $1 \mu \mathrm{s}$. При указанной задержке наблюдалось полное гашение на обеих линиях (рис. $2, b$ ).

Энергия импульса предионизации варьировалась путем изменения напряжения, до которого заряжается емкость $C 2$. Видно, что подавление на линии $510.6 \mathrm{~nm}$ наблюдается при энергии, запасаемой в конденсаторе $C 2$ $(\sim 3.5 \mathrm{~mJ})$. Для полного подавления излучения на желтой линии требуется $5 \mathrm{~mJ}$. Данная особенность связана, на наш взгляд, с заселением нижних рабочих уровней.

Нами был разработан макет $\mathrm{CuBr}$-лазера большого объема с непрерывным управлением энергией каждого импульса излучения. Схема синхронизации и независимое изменение параметров импульсов возбуждения и предионизации позволяют детально исследовать процессы, протекающие в активном элементе. Отличительной особенностью является наличие водородсодержащей добавки $\mathrm{HBr}$ в активной среде, которая позволяет повысить частотно-энергетические параметры лазера. Кроме того, получены регулировочные характеристики для каждой спектральной линии. Показано, что подавление линии $510.6 \mathrm{~nm}$ происходит при меньших значениях энергии импульса предионизации $(3.3 \mathrm{~mJ})$, и управление осуществлялось в более широком диапазоне задержек (вплоть до $4 \mu \mathrm{s}$ ), чем для желтой линии. Для желтой линии $578.2 \mathrm{~nm}$ подавление возможно в диапазоне задержек от 0 до $2 \mu \mathrm{s}$ и требует большей энергии импульса предионизации $(5 \mathrm{~mJ})$. Разработанный макет будет использован для детального исследования механизмов, определяющих эффективность генерации и усиления оптических сигналов активными средами на парах галогенидов металлов. 


\section{Благодарности}

Авторы выражают благодарность сотрудникам Института оптики атмосферы СО РАН В.А. Димаки и В.В. Власову за помощь в проведении экспериментов, а также Г.С. Евтушенко за обсуждение результатов и интерес к работе.

\section{Финансирование работы}

Исследование возможностей оперативного управления характеристиками среды на самоограниченных переходах атомов металлов выполнено в рамках проекта Российского научного фонда № 19-79-10096, работа по повышению эффективности источников излучения видимого диапазона проведена в рамках ГЗ АAАA A17117021310150-0.

\section{Конфликт интересов}

Авторы заявляют, что у них нет конфликта интересов.

\section{Список литературы}

[1] Little C.E. Metal vapor lasers: physics, engineering \& applications. Chichester (UK): John Willey \& Sons Ltd., 1998. 620 p.

[2] Евтушенко Г.С., Казарян М.А., Торгаев С.Н., Тригуб М.В., Шиянов Д.В. Скоростные усилители яркости на индуцированных переходах в парах металлов. Монографическая серия. Томск: STT, 2016. В. 1. 245 с.

[3] Тригуб М.В., Шиянов Д.В., Суханов В.Б., Петухов Т.Д., Евтушенко Г.С. // Письма в ЖТФ. 2018. Т. 44. В. 24. C. 135-142. DOI: 10.21883/PJTF.2018.24.47041.17523

[4] Суханов В.Б., Шиянов Д.В., Тригуб М.В., Димаки В.А., Евтушенко Г.С. // Письма в ЖТФ. 2016. Т. 42. В. 6. C. 84-89.

[5] Солдатов А.Н., Федоров В.Ф. // Квантовая электроника. 1983. T. 10. № 5. C. 974-980.

[6] Федоров А.И., Федоров В.Ф., Димаки В.А. // Оптика атмосферы и океана. 2012. Т. 25. № 02. С. 198-202.

[7] Behrouzinia S., Salehinia D., Khorasani K., Farahmandjou M. // Opt. Commun. 2019. V. 436. P. 143-145. DOI: $10.1016 /$ j.optcom.2018.12.016

[8] Тригуб М.В., Евтушенко Г.С., Троицкий В.О. // Оптика атмосферы и океана. 2016. Т. 29. № 02. С. 162-164.

[9] Boichenko A.M., Evtushenko G.S., Nekhoroshev V.O., Shiyanov D.V., Torgaev S.N. // Phys. Wave Phenom. 2015. V. 23. N 1. P. 1-13.

[10] Батенин В.М., Бучанов В.В., Казарян М.А., Климовский И.И., Молодых Э.И. Лазеры на самоограниченных переходах атомов металлов. М.: Науч. кн., 1998. 544 с. 